\title{
Refractory Malignant Urinary System Neoplasm
}

National Cancer Institute

\section{Source}

National Cancer Institute. Refractory Malignant Urinary System Neoplasm. NCI

Thesaurus. Code C150543.

Malignant urinary system neoplasm that is resistant to treatment. 